\title{
Are we aware of what our patients eat?
}

\author{
Motives underlying food choice: dentists, porters and dietary health promotion by M. L. Crossley and S. N. Khan \\ Br Dent J 2001; 191: 198-202
}

\begin{abstract}
Objective
Differences in dental decay and disease amongst socioeconomic groups are thought to derive, in part, from variations in dietary practices and differences in education. The aim of this exploratory study was to examine whether differences in motivating factors affecting food choice could be found in a comparison of two groups at very different ends of the social spectrum: dentists and porters/cleaners.
\end{abstract}

\section{Design}

A convenience sample of 100 people (51 porters/cleaners and 49 dentists) working in the dental school at a university in the North West of England were approached to interview face-to-face and complete the Food Choice Questionnaire (FCQ), a previously validated measure designed to assess nine main factors relevant to peoples' food choices. A sample size of 100 was chosen because it was adequate to test validity (using a two-group Chi-square test with a 0.050 two sided significance).

\section{Results}

Findings were analysed using independent sample $t$-test and multiple linear regression. Results indicated significant differences between porters/cleaners and dentists in terms of their motives for food choice on six of the nine FCQ factors. These included convenience $(\mathrm{p}<0.001)$, natural content $(\mathrm{p}<0.05)$, price $(\mathrm{p}<0.005)$, familiarity $(\mathrm{p}<.0001)$, mood $(\mathrm{p}<0.03)$ and ethical concern $(\mathrm{p}<0.01)$. Porters/cleaners tended to rate the factors covenience, price, mood and familiarity more highly, whereas dentists did the same for natural content and ethical concern.

\section{Conclusions}

Awareness of the differences in motivational factors affecting food choice between different social groups is important to dental practitioners who are being taught to play an increasing role in health promotion. If dental practitioners are to partake meaningfully in such a role, it is necessary for them to be aware not only of their own motives in food selection, but also of the way in which those motives may differ from those of their clients.

\author{
In Brief \\ - Are there differences in the motivating factors affecting \\ food choice between two groups at opposite ends of the \\ social spectrum? \\ - The Food Choice Questionnaire was distributed to a \\ convenience sample of dentists and porters/cleaners \\ - Compared with porters/cleaners, dentists were more likely \\ to choose food in terms of motives relating to 'natural \\ content' and 'ethical concern' \\ - Compared with dentists, porters/cleaners were more likely \\ to choose food in terms of convenience, price, mood and \\ familiarity motives \\ - Awareness of the differences in motivational factors \\ affecting food choice is important for dentists in terms of \\ their increasing role in health promotion.
}

\section{Comment}

$W^{\text {hilst all dentists know that diet plays a }}$ key role in causing dental caries, little is known about why people choose the foods they eat. For a dentist wishing to influence a patient's diet this paucity of information poses a problem, as it is very difficult to motivate someone to change, if you have no understanding of what influences their behaviour. This paper seeks to rectify this deficiency, specifically in the dental context, by using an instrument called the Food Choice Questionnaire. This questionnaire has 36 questions, which relate to 9 factors, which appear to influence what people choose to eat.

By administering the questionnaire to a group of dentists and a group of porters and cleaners in a university, the paper examines differences between the two groups on food choices. The paper argues powerfully that it is helpful for dentists to understand the issues underlying what they themselves eat, in order to better understand their patients' behaviours. This idea, that self awareness leads to increased professional competence, is an appealing one and indicates that the psyche of dentists might offer fertile ground for research!

The results of the study showed that the porters/cleaners (these occupations being used as proxy indicators of low socio-economic status) chose their foods in relation to price, how familiar the food was and what mood the individual was in when making their choices. By contrast, the dentists claimed that their food choices were driven by concern about ethics and about the 'naturalness' of the food. What food looks, smells and tastes like and how convenient it is to prepare and eat were important motivators of choice for both groups.

The paper is perhaps a salient reminder to us that as dentists we are extremely privileged and fortunate. We therefore simply do not have, and therefore cannot fully understand, some of the very real concerns of those whom we treat and offer choices to. Indeed, the dentist who reported in the study that he/she could 'eat out every night' clearly inhabits a very different world to most working class people. This must make it very difficult for him/her to truly sympathise or empathise with others whose choices about food are driven by real economic hardship.

This paper offers insights into ourselves, and an understanding of differences between dentists and others which are perhaps as important as the interesting scientific findings which it presents.

\section{Elizabeth J Kay}

Professor of Dental Health Services Research,

University of Manchester Dental School 